EPiC Series in Computing
Volume 58, 2019, Pages 407-417
Proceedings of 34th International Confer-
ence on Computers and Their Applications

\title{
Finite Element Analysis of 3D Printed Aircraft Wing
}

\author{
Julian Rogers ${ }^{1}$ and Hormoz Zareh ${ }^{2}$ \\ Mechanical and Materials Engineering Department, Portland State University, 97201, USA \\ ${ }^{1}$ Research student. jurogers@pdx.edu \\ ${ }^{2}$ Associate professor. hormoz@pdx.edu
}

\begin{abstract}
3D printing has allowed complex designs to be produced that were impossible to create using conventional manufacturing processes. Aircraft wings are optimized as much as possible given manufacturability considerations, but more complex geometry could provide the same strength for less weight, increasing aircraft performance. Although carbon fiber composites are some of the best known materials for conventional optimized aircraft wings, current 3D printing technology cannot produce this material. Instead, it is currently limited to metals and polymers. To determine if the more complex geometry which can be produced by 3D printing can offset the material limitations, a carbon fiber composite wing and a redesigned, 3D printed 7075-T6 aluminum wing were compared using Finite Element Analysis. The unoptimized 3D printed aluminum wing had a superior safety factor against fracture/yielding (1,109\% higher) and buckling resistance (127.3\% higher), but at the cost of a $23.99 \%$ mass increase compared to the optimized carbon fiber composite wing. If the $3 \mathrm{D}$ printed aluminum wing had been optimized to provide the same safety factor against fracture/yielding and buckling resistance as the carbon fiber composite wing, it is anticipated that the resulting design would be significantly lighter, thus increasing aircraft performance.
\end{abstract}

\section{Introduction}

Designing aircraft is a never-ending pursuit of lighter and stronger designs, allowing for higher speeds, ranges, and efficiencies. Three main material types have been used in the of aviation: wood and fabric [1], metals [2], and composites [3]. The original 1903 Wright Flyer used a wooden frame with a fabric covering, and this construction method continued into the 1920s. At this time, designers started using metals for structural components, as they allowed for much stronger parts. Metals are still used in many aircraft today, although composites are starting to be used more often. Composites, which are a combination of two dissimilar materials, promise increased strength with lower mass compared to 
metals. These usually consist of a fiber material - commonly carbon, although boron and glass have also been used - in a polymer matrix. The first composite aircraft flew in 1969 [4], although the recently introduced Boeing 787 is the first airliner to make extensive use of the material type [5].

An additional method to increase the strength and decrease the mass of aircraft components comes from optimizing the internal structure. Wings are typically made with an exterior skin in the shape of an airfoil, which resists shear loadings and generates lift. Spars running from the wing root to tip carry the main bending and shear loads from the lift force, while ribs help form the skin shape and prevent it from buckling [6]. These parts are shown and labeled in Figure-1.

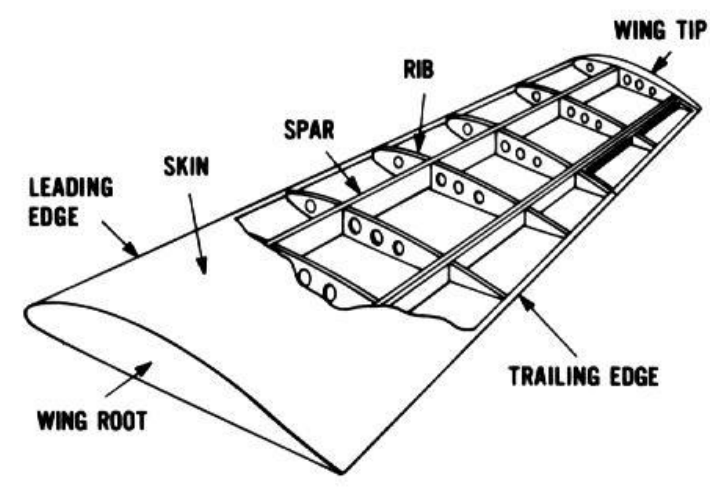

Figure-1: Typical wing design with skin, spar, and rib labeled [7].

This design is widely used in aircraft wings as it provides a good strength to mass ratio while still being relatively easy to manufacture. Although more complicated geometries can provide higher strength for the same mass, they are difficult, and sometimes even impossible, to manufacturing using traditional methods such as machining, welding, or forming.

The advent of Additive Manufacturing removes this design restriction. One of the most common Additive Manufacturing processes is $3 \mathrm{D}$ printing. There are several subcategories of $3 \mathrm{D}$ printing, but they all work on the same principle: Individual layers of material are selectively fused together to create the final part. Unlike with traditional manufacturing, where complexity leads to increased costs, 3D printing is not affected by part geometry. It is just as easy and time consuming to print a simple cube as it is to create a complex lattice structure. This allows for more complicated geometry which was previously impractical or downright impossible to produce.

While 3D printing provides many advantages with regards to part complexity, it cannot match the material selection of traditional manufacturing methods. Currently there is no way to print a carbon fiber composite part with the same strength as a part with a traditional layup. Instead, 3D printing is currently used with polymers and metals. Printable materials include 7075 aluminum [8] and grade 5 titanium [9], both of which are useful for aircraft parts.

To determine if the complex geometry enabled by 3D printing can overcome the material shortcomings, two wings were analyzed using Abaqus Finite Element Analysis (FEA) software. The first model was a copy of a carbon fiber composite wing designed and optimized for a High Altitude, Long Endurance (HALE) aircraft [6]. This was then compared to a proposed 3D printed wing which used the same skin geometry but had an internal structure inspired by 3D Lab Print's model aircraft [10]. Two views of the company's Spitfire MK XVI RC aircraft and internal wing structure are shown in Figure-2 and Figure-3. This is one of the first model RC aircraft designed for 3D printing. 


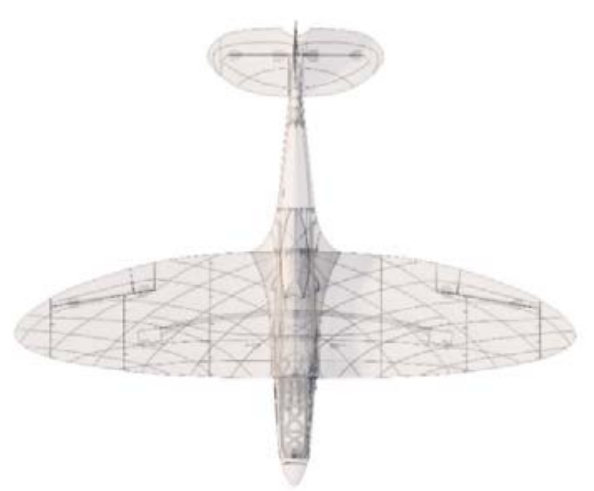

Figure-2: Top view of 3D Lab Print's Spitfire MK XVI showing curved ribs/spars [10].

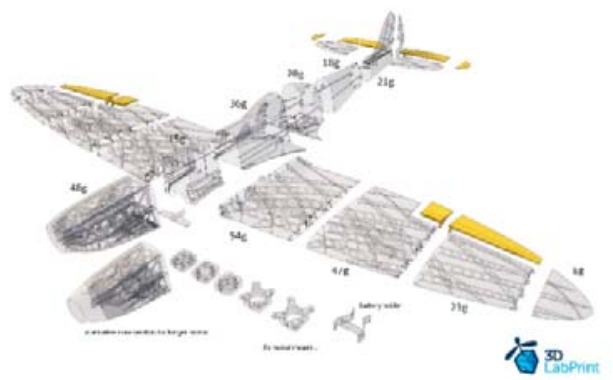

Figure-3: Isometric view of Spitfire MK XVI showing lightening holes in wing ribs/spars [10].

\section{Wing Designs}

\section{Carbon Fiber Composite}

To provide a baseline design, a carbon fiber wing was constructed based on a carbon fiber composite HALE aircraft wing. The airfoil used was a Wortmann FX 63 137. The wing was a two-piece design, with the outer section swept back $4.7^{\circ}$. A compilation of the wing skin design variables is in Table-1, while Figure-4 shows the relevant sections and measurements [6].

\begin{tabular}{|c|c|c|}
\hline Property & Inboard & Outboard \\
\hline Half Span & $9.7 \mathrm{~m}$ & $5.4 \mathrm{~m}$ \\
\hline Root Chord $(\mathrm{Cr})$ & $2.138 \mathrm{~m}$ & $1.283 \mathrm{~m}$ \\
\hline Tip Chord $(\mathrm{Ct})$ & $1.283 \mathrm{~m}$ & $0.472 \mathrm{~m}$ \\
\hline Leading Edge Sweep & $0^{\circ}$ & $4.7^{\circ}$ \\
\hline
\end{tabular}

Table-1: Design dimensions for Wortmann wing design [6]. 


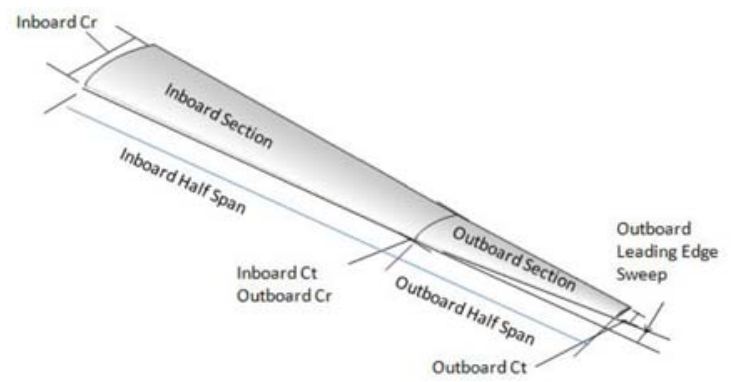

Figure-4: Visual representation of wing skin design variables [6].

For the carbon fiber composite wing, the design contained three spars at the $15 \%, 45 \%$, and $60 \%$ chords. Additionally, there were 59 ribs spaced at $250 \mathrm{~mm}$ intervals from the wing root. Finally, the wing skin in front of the $15 \%$ spar and behind the $60 \%$ spar was removed, leaving only the wingbox, which takes all the flight loading.

There were nine different composite layups used in the wing. The wing was broken into three sections: wing root to $16^{\text {th }}$ rib, $16^{\text {th }}$ rib to half span $(9.7 \mathrm{~m})$, and half span to wing tip. Additionally, the skin, spars, and ribs had different thicknesses and ply orientations, resulting in nine distinct, symmetric layups. For each layup, one to three laminae of unidirectional carbon fiber composite were used for the cover, with an aramid honeycomb core. The thicknesses and orientations of each ply were taken from the original analysis [6]. The material properties used for the unidirectional carbon fiber are in Table2 , while the properties used for the aramid honeycomb core are in Table-3. Both of these were entered as laminae in Abaqus.

\begin{tabular}{|c|c|}
\hline Density $(\rho)$ & $1,600 \mathrm{~kg} / \mathrm{m}^{3}$ \\
\hline Young's Modulus $0^{\circ}(\mathrm{E} 1)$ & $135 \mathrm{GPa}$ \\
\hline Young's Modulus 90 $0^{\circ}(\mathrm{E} 2)$ & $10 \mathrm{GPa}$ \\
\hline Major Poisson's Ratio (Nu12) & 0.30 \\
\hline Shear Modulus 12 (G12) & $5 \mathrm{GPa}$ \\
\hline Shear Modulus 13 (G13) & $5 \mathrm{GPa}$ \\
\hline Shear Modulus 23 (G23) & $5 \mathrm{GPa}$ \\
\hline
\end{tabular}

Table-2: List of carbon fiber composite material properties used [11].

\begin{tabular}{|c|c|}
\hline Density $(\rho)$ & $200 \mathrm{~kg} / \mathrm{m}^{3}$ \\
\hline Young's Modulus $0^{\circ}(\mathrm{E} 1)$ & $70 \mathrm{kPa}$ \\
\hline Young's Modulus 90 $(\mathrm{E} 2)$ & $70 \mathrm{kPa}$ \\
\hline Major Poisson's Ratio (Nu12) & 0.0 \\
\hline Shear Modulus 12 (G12) & $14 \mathrm{MPa}$ \\
\hline Shear Modulus 13 (G13) & $14 \mathrm{MPa}$ \\
\hline Shear Modulus 23(G23) & $21 \mathrm{MPa}$ \\
\hline
\end{tabular}

Table-3: List of aramid honeycomb core material properties used [12]. 
A view of the final model of the carbon fiber composite wing used for the FEA study is shown in Figure-5.

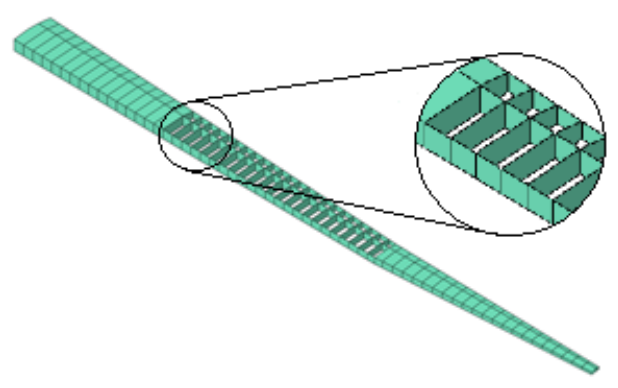

Figure-5: Carbon fiber composite FEA model.

\section{D Printed Aluminum}

For the 3D printed wing, the front (15\%) spar, rear (60\%) spar, and wing skin geometry were retained, while the middle (45\%) spar and ribs were removed. These were replaced by curved members created by two sets of concentric circles. A top view of the internal geometry is shown in Figure-6. Additionally, lightening holes were cut in all of the spars and curved members. These holes were sized such that they were $60 \%$ of the height of the member at the center of the circle, and evenly spaced along the member. The spaces between the edges of consecutive holes were approximately $50 \mathrm{~mm}$ (the holes were evenly spaced along the member). A view of the lightening holes is shown in Figure-7. These holes were added as they significantly reduce the mass of the structure without sacrificing strength, as the center section of a beam carries much less loading than material far from the neutral axis.

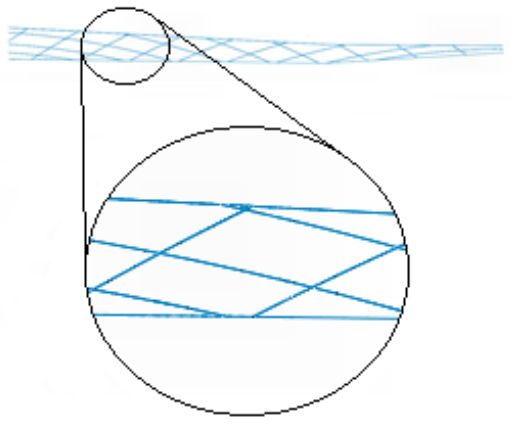

Figure-6: Top view of curved internal members. Outer skins have been removed for clarity.

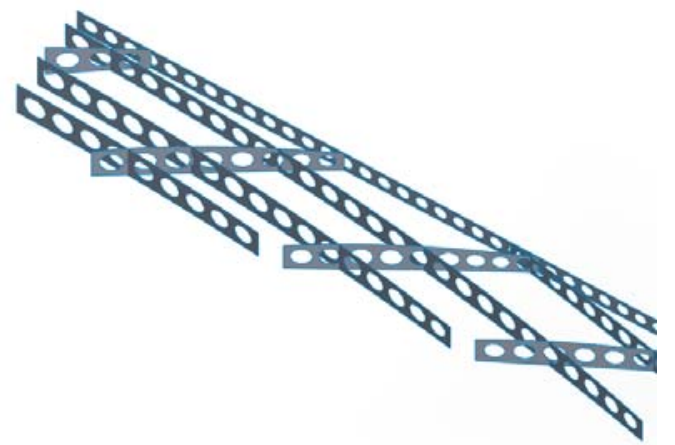

Figure-7: Lightening holes in the internal structure of the 3D printed wing model. 
A variable thickness was defined for the spars, internal structure, and wing skin. This was to better utilize the strength of the structural material. Since lift is distributed along the wing, the greatest shear and bending load occur at the wing root, decreasing to 0 at the wing tip. If the structural members are the same thickness along the wing span, the material at the wing tips has less loading than the material at the wing root. This means that the structure at the wing tip and along the wing span can be significantly lightened before failing, resulting in more of the wing material being fully stressed and a lower total mass. While conventional manufacturing makes variable thickness structural members impractical, 3D printing allows for an optimized design such as this. For this analysis, the member thicknesses varied linearly from root to tip. The root and tip thicknesses used in the final models are shown in Table-4.

\begin{tabular}{|c|c|c|}
\hline Region & Root & Tip \\
\hline Spars/Internal Structure & $1.75 \mathrm{~mm}$ & $0.5 \mathrm{~mm}$ \\
\hline Skin & $5.5 \mathrm{~mm}$ & $0.5 \mathrm{~mm}$ \\
\hline
\end{tabular}

Table-4: Wing root and tip thicknesses for 3D printed structural members. The thicknesses vary linearly from the root to the tip.

Finally, 7075-T6 aluminum was chosen as the wing material. This grade of aluminum is typically used in aircraft and has a high strength-to-mass ratio. It can also be 3D printed [8]. The material properties used for the analysis are in Table-5. The material was defined as uniform and isotropic in Abaqus.

\begin{tabular}{|c|c|}
\hline Density $(\rho)$ & $2,810 \mathrm{~kg} / \mathrm{m}^{3}$ \\
\hline Young's Modulus $(\mathrm{E})$ & $71.1 \mathrm{GPa}$ \\
\hline Poisson's Ratio $(v)$ & 0.33 \\
\hline
\end{tabular}

Table-5: Material properties for 7075-T6 aluminum used in 3D printed wing [13]

\section{Finite Element Analysis}

Abaqus FEA software was used to analyze both wing designs. The two main failure modes of interest were buckling and material fracture or yielding. In order to capture both failure types, a linear buckling and a static linear analysis were conducted. The buckling analysis used the Lanczos Eigen solver to determine buckling modes and critical loads. The static linear analysis was performed with a single increment as large nonlinear deformations were expected. As a validation, a geometric nonlinear analysis for each model was run which deviated from the linear models by less than $0.25 \%$ for all relevant variables of interest, indicating no nonlinear effects.

As the two wing models had different geometries, they required two different formulations of shell elements. For the carbon fiber composite model, the regularity of the geometry allowed S4, four node, linear quadrilateral shell elements to be used with a sweep meshing structure. The 3D printed wing model had more irregular geometry which required STRI65, six node, quadratic incompatible triangle shell elements with a free meshing scheme.

For both models, a convergence study was run to verify the accuracy of the results. The meshes were refined by varying the global mesh seed sizes. The element sizes were determined by the number of equally sized elements between each rib in the carbon fiber composite wing. The mesh started with two elements in the $250 \mathrm{~mm}$ span between consecutive ribs and increased by one element per span until 
convergence had been achieved. These mesh seed sizes were also used for the 3D printed aluminum wing, although they did not correspond to the number of elements between consecutive ribs. Convergence was considered to be achieved when the percentage difference between the current and previous models was less than $1 \%$ for the von Mises stress at the point of interest, first positive buckling eigenvalue, and maximum deflection. Figure- 8 shows the convergence graph for the carbon fiber composite wing while the convergence graph for the 3D printed aluminum wing is shown in Figure-9.

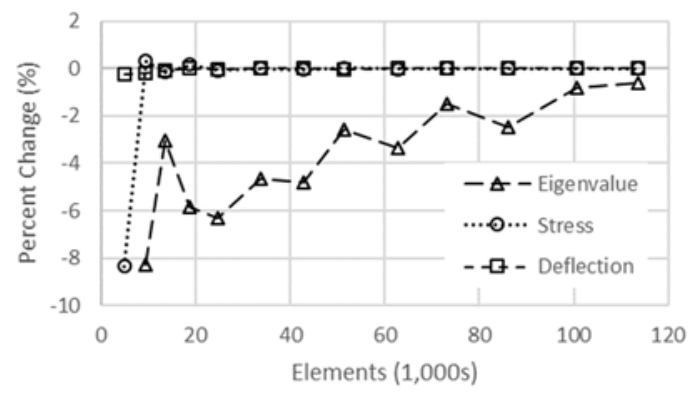

Figure-8: Convergence graph for carbon fiber composite wing with first positive buckling eigenvalue, von Mises stress, and tip deflection.

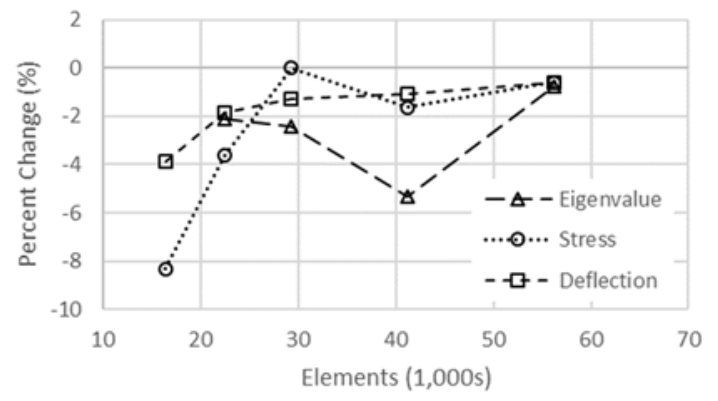

Figure-9: Convergence graph for 3D printed aluminum wing with first positive buckling eigenvalue, von Mises stress, and tip deflection.

The carbon fiber composite wing model converged with 100,647 elements, while the 3D printed aluminum wing converged with 56,193 elements. The quadratic triangular shells converged with fewer elements than the linear quadrilateral shells, as expected.

Since this is a comparative study between two different design philosophies, the loading and boundary conditions need to be representative of real world conditions, but are not required to exactly match the real world loading so long as they are identical for both models. In order to replicate a realistic lift distribution, an elliptical pressure distribution was defined, varying from $1000 \mathrm{~Pa}$ at the wing root to 0 $\mathrm{Pa}$ at the wing tip. The equation used to define this distribution is given in Equation 1, where $P$ is the pressure in Pascals and $x$ is the span-wise coordinate in meters, starting at $0 \mathrm{~m}$ at the wing root and extending to $15.133 \mathrm{~m}$ at the wing tip. There was no variation in the pressure distribution between the leading and trailing edges. This pressure load was applied to the lower skin surface. The pressure distribution is shown in Figure-10. Additionally, a gravity force of $9.81 \mathrm{~m} / \mathrm{s}^{2}$ was applied to the models. 


$$
P=1000 * \sqrt{1-\frac{x^{2}}{15.133^{2}}}
$$

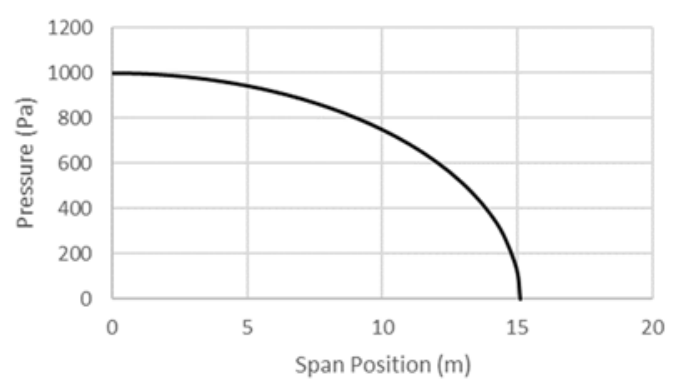

Figure-10: Graphical representation of pressure load applied to lower wing skin.

The boundary conditions simulated a standard cantilever wing attachment to a fuselage. All of the shell edges along the wing root were restrained in all six D.O.F.s. This was required as shell elements have three translational and three rotational degrees of freedom.

\section{Results and Discussion}

The carbon fiber composite model was intended to replicate an HALE aircraft wing and to provide a reference for the $3 \mathrm{D}$ printed aluminum wing. The exact material properties and loadings used were not specified in the original analysis [6], thus the carbon fiber composite design for this study was also necessary to provide an accurate comparison of the two designs. The original analysis used the TsaiWu failure criteria to determine whether the wing material had fractured. Abaqus does not natively provide Tsai-Wu failure criteria; it was assumed that the results of the original analysis were valid. The first positive buckling eigenvalue and wing tip deflection can be directly correlated between the two models and are summarized in Table-6.

\begin{tabular}{|c|c|c|}
\hline Property & Original & Current \\
\hline Buckling Eigenvalue & 1.102 & 1.075 \\
\hline Tip Deflection & $1,206 \mathrm{~mm}$ & $271 \mathrm{~mm}$ \\
\hline
\end{tabular}

Table-6: Comparison of FEA results from original analysis [6] and current carbon fiber composite wing

The first positive bucking eigenvalue for the carbon fiber composite wing model correlates well with the original analysis, especially considering that was based on a full Computational Fluid Dynamics (CFD) simulation and the load for this analysis was a simple approximation. The tip deflection is significantly different between the two analyses though. The original analysis reported a tip deflection of $1,206 \mathrm{~mm}$, while this model reported $271 \mathrm{~mm}$.

This discrepancy could be due to several factors. The exact material properties used in the original analysis were not specified. A reference for unidirectional carbon fiber composite was given in the paper, but the failure code for the Tsai-Wu criteria used ultimate strength values that were exactly $50 \%$ of the ones specified from that source [6][11]. No safety factor or explanation for this was mentioned in the original analysis. Thus, the elastic material properties could also have been reduced by one half, leading to higher tip deflections. Additionally, no properties or source were specified for the honeycomb material, so representative elastic properties for aramid honeycomb were used [12], while 
the density was adjusted so the model's mass matched the mass from the original analysis. This could also affect the results, although it would have a smaller effect since the honeycomb material's stiffness contribution was very low compared to the unidirectional carbon fiber composite. Finally, the pressure distribution for this model was based on an assumed elliptical span-wise lift distribution. While this is a good approximation, it does not take into account the exact flow characteristics of specific wings. Thus, the CFD data from the original analysis would be a better representation of the real loads applied to the wing. Fortunately, since this analysis is comparing the relative performance of two designs and not attempting to design a wing to be used on a real aircraft, as long as the loadings are identical between the carbon fiber composite and 3D printed aluminum wing, loading inaccuracies can be ignored.

For the comparison between the carbon fiber composite wing and the 3D printed wing, the main variables that were compared were the mass, fracture or yielding safety factor, first positive buckling eigenvalue, and tip deflection. The results from the two analyses are summarized in Table- 7 .

\begin{tabular}{|c|c|c|}
\hline Property & Carbon Fiber & 3D Aluminum \\
\hline Mass & $175.9 \mathrm{~kg}$ & $218.1 \mathrm{~kg}$ \\
\hline $\begin{array}{c}\text { Fracture/Yielding } \\
\text { Safety Factor }\end{array}$ & 1.063 & 12.85 \\
\hline Buckling Eigenvalue & 1.075 & 2.444 \\
\hline Tip Deflection & $270.6 \mathrm{~mm}$ & $260.0 \mathrm{~mm}$ \\
\hline
\end{tabular}

Table-7: Mass, safety factor, and first positive buckling eigenvalue for carbon fiber composite and 3D printed aluminum wing. The fracture safety factor for the carbon fiber wing is taken from the original analysis [6]; all other values are from this analysis.

The 3D printed aluminum wing shows superior fracture/yielding safety factor (1,109\% higher), buckling resistance (127.3\% higher), with a lower tip deflection (3.917\% lower). This does come at the cost of a $42.2 \mathrm{~kg}(23.99 \%)$ mass increase. However, this was achieved with an unoptimized geometry. The spar/rib shapes and spacings were designed to mimic the internal structure of 3D printed model aircraft [10], however there were no calculations to determine the optimum geometry. Additionally, the spar/rib and skin thicknesses were manually iterated less than ten times to provide a feasible wing. The unoptimized carbon fiber composite wing from the original analysis had a higher mass $(251 \mathrm{~kg})$, and lower failure factors (fracture safety factor of 1.605 and critical buckling eigenvalue of 1.35) than the corresponding unoptimized 3D printed aluminum wing [6]. Running an optimization algorithm for both the internal geometry as well as the thickness of each member to lower the failure indices to those specified in the original analysis ( $>1$ for yielding safety factor and $>1.1$ for buckling eigenvalue) would be able to create a 3D printed aluminum wing with the same strength as a carbon fiber composite wing but with a reduced mass, improving aircraft performance [6].

The stress distribution for the 3D printed aluminum wing is also more uniform than the carbon fiber composite wing. For an efficient structure, most parts of the wing should be equally stressed. Lower stressed areas indicate excess strength which is unused, as the higher stressed areas will fail first. As the old adage states, a chain is only as strong as its weakest link. The Von Mises stress distributions are shown in Figure-11 and in Figure-12. 


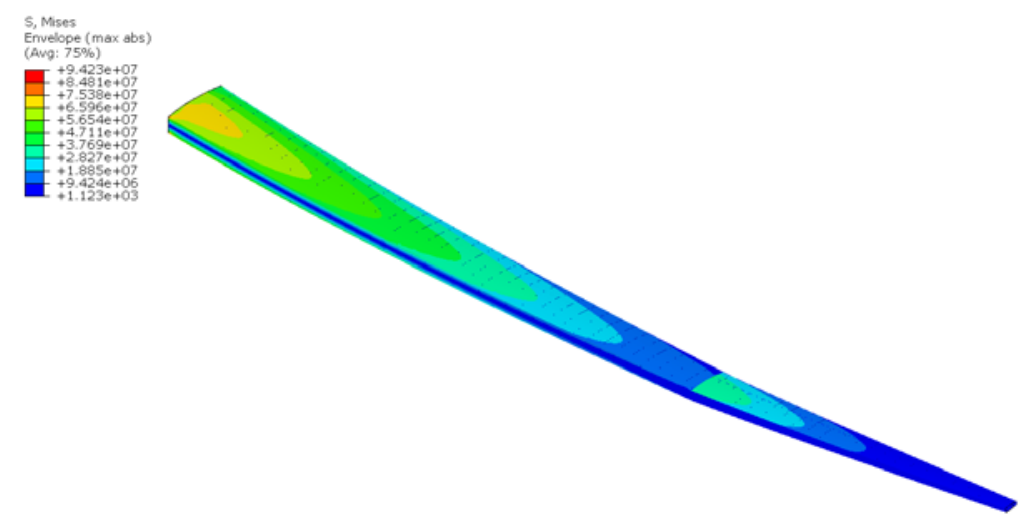

Figure-11: von Mises stress distribution for carbon fiber composite wing. There is a high stress area near the wing root. There is also a higher stressed area in the outboard section of the wing due to thinner plies.

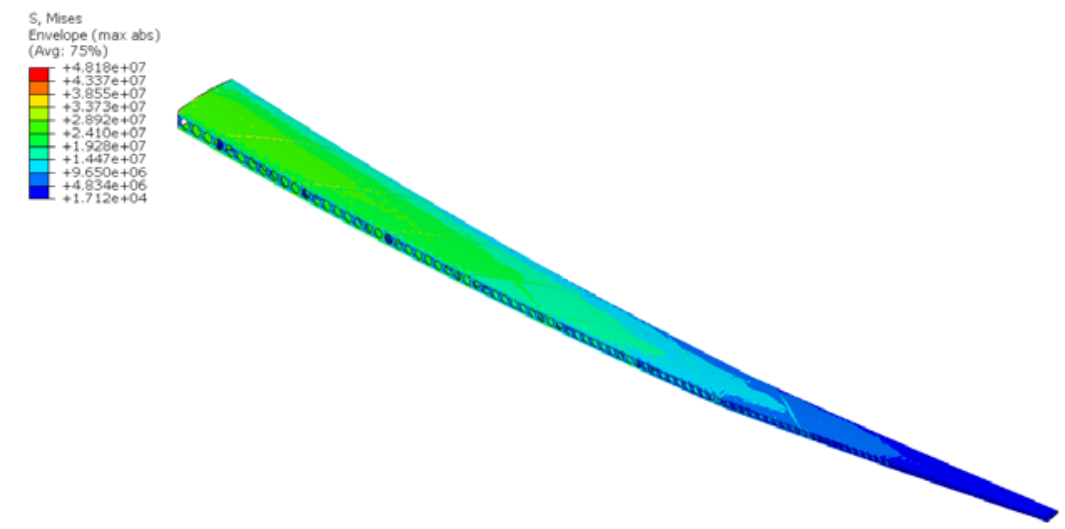

Figure-12: von Mises stress plot for 3D printed aluminum wing. Stress is fairly evenly distributed between the wing root and mid-span.

The carbon fiber composite wing has a high stress area in the top skin near the wing root. The rest of the model is not stressed as highly, which indicates that the rest of the structure is stronger than it needs to be. The outer section of the wing was made thinner which resulted in a second high stress area. This was done to reduce the overall mass of the wing, since the high strength required at the wing root was not required at the wing tip since it experiences lower loads. Conversely, the 3D printed aluminum wing has a much more uniform stress distribution. This is due to the continuously varying skin and spar/rib thickness, which results in a better optimized structure.

\section{Conclusions}

This analysis has shown that the increased complexity which can be produced by 3D printing aluminum can offset the material deficiency compared to carbon fiber composite in an aircraft wing. Although these preliminary results did not result in a 3D printed aluminum wing which had a lower mass than an optimized carbon fiber composite wing, the strength and failure indices were significantly higher, indicating that optimizing the $3 \mathrm{D}$ printed wing would result in a design superior to the carbon fiber composite wing. Additionally, using grade 5 titanium or other metals to construct the wing could 
be explored, as optimized structures using different materials could be superior to the 7075-T6 aluminum design used for this analysis. Finally, this design was based on conventional planar geometries. Even though the curved spars/ribs are more complex and optimized than a traditional spar and rib design, they are not necessarily the best solution. Topology optimization can help design a part that is completely optimized and equally stressed throughout. Previously, these designs were too complex to produce through conventional means, as they usually contain intricate curved geometry which was impossible fabricate by machining, forming, or welding. 3D printing has no such limitation, and can create parts such as those with ease. Thus, future work could focus on analyzing a wing that has been topologically optimized to determine such a design is better optimized.

\section{References}

[1] Mraz, S., 2003, "A century of progress in aircraft materials - Part 1 Wood and Fabric," Machine Design [Online]. Available: http://www.machinedesign.com/archive/century-progress-aircraftmaterials-part-1-wood-and-fabric. [Accessed: 12-May-2018].

[2] Mraz, S., 2003, "A century of progress in aircraft materials - Part 2 Metals," Machine Design [Online]. Available: http://www.machinedesign.com/news/century-progress-aircraft-materialspart-2-metals. [Accessed: 12-May-2018].

[3] Mraz, S., 2003, "A century of progress in aircraft materials - Part 3 Composites," Machine Design [Online]. Available: http://www.machinedesign.com/archive/century-progress-aircraft-materialspart-3-composites. [Accessed: 12-May-2018].

[4] Garvey, W., 2015, "First All-Composite Aircraft Being Readied To Fly Again," Aviation Week [Online]. Available: http://aviationweek.com/business-aviation/first-all-composite-aircraft-beingreadied-fly-again. [Accessed: 12-May-2018].

[5] Molnar, M., 2009, "Boeing 787 Dreamliner Finally Gets Off the Ground," Popular Mechanics [Online]. Available: https://www.popularmechanics.com/flight/a5255/4339887/. [Accessed: 12May-2018].

[6] Arévalo Philip T., 2014, "Design Optimization of a Composite Wing Box for High-Altitude Long-Endurance Aircraft," thesis, Embry-Riddle Aeronautical University.

[7] 2014, "Stress Analysis in Wing and Fuselage," Available: https://pritamashutosh.wordpress.com/2014/04/23/stress-analysis-in-wing-and-fuselage/. [Accessed: 12-May-2018].

[8] “3D Printed 60617075 Aluminum," RE3DTECH [Online]. http://www.re3dtech.com/3dcomposites/3d-printed-6061-7075-aluminum/. [Accessed: 12-May-2018].

[9] “3D Printing Material: Titanium 6AI-4V," Sculpteo [Online]. Available: https://www.sculpteo.com/en/materials/metals/dmls-titanium-material/.

[10] "Spitfire Mk XVI," 3D Lab Print [Online]. Available: https://3dlabprint.com/shop/spitfire-mkxvi-s3d-cura-ready/. [Accessed: 12-May-2018].

[11] "Mechanical Properties of Carbon Fibre Composite Materials, Fibre / Epoxy resin $\left(120^{\circ} \mathrm{C}\right.$ Cure)," Performance Composites [Online]. Available: http://www.performancecomposites.com/carbonfibre/mechanicalproperties_2.asp. [Accessed: 12-May-2018].

[12] Zareh, H., 2018, "Composite FEA for Abaqus."

[13] "Aluminum 7075-T6; 7075-T651," ASM Aerospace Specification Metals, Inc. [Online]. Available: http://asm.matweb.com/search/SpecificMaterial.asp?bassnum=MA7075T6. [Accessed: 12-May-2018]. 